\title{
Research and Demonstration of the Refraction Problems
}

\author{
Xiao Hu* , Lintong Zhang, Jixun Chu \\ School of Mathematics and Physics, University of Science \& Technology Beijing, Beijing, China \\ Email address: \\ 445342098@qq.com (Xiao Hu), 17888803421@163.com (Lintong Zhang), chujixun@ustb.edu.cn (Jixun Chu) \\ ${ }^{*}$ Corresponding author
}

\section{To cite this article:}

Xiao Hu, Lintong Zhang, Jixun Chu. Research and Demonstration of the Refraction Problems. American Journal of Applied Mathematics. Vol. 6, No. 2, 2018, pp. 87-96. doi: 10.11648/j.ajam.20180602.19

Received: May 10, 2018; Accepted: June 19, 2018; Published: June 26, 2018

\begin{abstract}
The introduction of function extremum promotes the development of calculus, which is the prerequisite and important condition for the development and development of many mathematical ideas. Except for extreme problems in social life or science and technology, the issue of cost in economic issues, the shortest distance in mathematics problem can be solved by the function extreme value thought. However, because the extreme value problem is not well described, learners cannot easily observe and learn. This paper takes the uniform speed and uniform acceleration and descent in the descent model as an example. In the uniform acceleration, the dichotomy is introduced to solve the function extremum problem. Through MATLAB simulation, the position of the steepest descent point is found intuitively and relevant conclusions are obtained. It is hoped that the research process of the problem can be helpful to the study of refractive problems, and it is hoped that the visually intuitive simulation results can enable learners to understand the descent model more objectively.
\end{abstract}

Keywords: Refractive Problems, Function Extremum, Dichotomy, MATLAB Simulation

\section{Introduction}

In mathematical analysis, the maximum and minimum values of a function within a given range are collectively referred to as extreme values [1]. Pierre de Fermat was one of the first mathematicians to find the function's maximum and minimum. The mathematical ideas and methods based on the one-element extremum problem are applied in many aspects and can be combined with optimization methods to solve optimization problems [2], solve precipitation problems [3] [4], rocket orbit problems [5], artificial intelligence problems [6], especially on the issue of the shortest time, this article can discuss the shortest time path problem of transportation [7] [8] [9], traffic problem [10], artificial intelligence problem [11], etc. Extreme values can be used in life, and all aspects of learning. Extremum problems are also more extensive solutions, the use of physical methods [12], mathematical methods [13] and so on. MATLAB is one of the key tools for the analysis of modern mathematics and physics problems. It has a very important supporting role in physics teaching [14] [15] [16] and mathematics teaching [17] [18].

This paper starts from the concept and definition of the one-variable function extremum, and discusses the shortest time based on uniform acceleration and descent. Gradually derive the calculation method of time and distance, at the same time, organically combine dichotomy ideas, and finally launch relevant conclusions. Finally, using MATLAB tools for simulation, the description of the problem and the final result are all more intuitive, which will greatly help to understand the refraction problem in the shortest time.

\section{One-Way Function Extreme Value Theory}

\subsection{Definition}

Let function $f(x)$ be defined in interval $(a, b)$, $x_{0} \in(a, b)$, if in $x_{0}$, a certain neighborhood of the mind has: $f(x) \leq f\left(x_{0}\right) \quad$ (or $f(x) \geq f\left(x_{0}\right)$ ), then $f\left(x_{0}\right)$ is a maximum (or a minimum) of function $f(x)$, and $x_{0}$ is a maximum (or minimum) point of $f(x)$. The maxima and minima are collectively referred to as the extremum of the function, and the maxima and minima are collectively referred to as extremum points of the function. 


\subsection{Steps}

The steps for finding the extremum of a one-way function are:

(1) Determine the domain of the function;

(2) Let $f^{\prime}(x)=0$, find all the stagnation points, examine the symbols around each stagnation point, and take the opposite extreme, otherwise do not take the extreme value;

(3) If $f^{\prime}\left(x_{0}\right)=0, f^{\prime \prime}\left(x_{0}\right) \neq 0$. When $f^{\prime \prime}\left(x_{0}\right)<0, f(x)$ takes a maximum value in $x_{0}$, when $f^{\prime \prime}\left(x_{0}\right)>0, f(x)$ takes a minimum value in $x_{0}$;

(4) Find the function value at each extreme point to get the corresponding extreme value using the above steps; you can solve extreme problems in real life.

\section{The Shortest Time Problem of Constant Speed Drop}

\subsection{Problem Description}

The particle moves from point $B(0, b)$ to point $A(a, 0)$, the speed of line $B D$ is $v_{1}$, and the speed of line $D A$ is $v_{2}$. Find the horizontal axis $x$ of the point $D\left(x, y_{0}\right)$ on the horizontal line $y=y_{0}$, so that the time from $B$ to $D$ reaches $A$ is the shortest, where the speeds $v_{1}$ and $v_{2}$ are known quantities, the specific problem is shown in the figure:

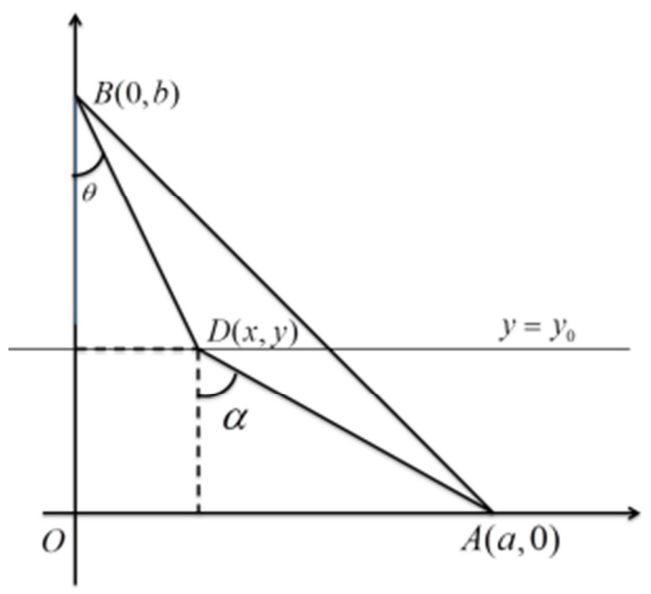

Figure 1. The description of shortest time problem of constant speed drop.

\subsection{Basic Solution}

\subsubsection{Time}

In order to obtain the coordinates of the most advantage $D$, the whole process is divided into two sections: $y_{0} \leq y \leq b$ and $0 \leq y<y_{0}$. From the physical principles and the Pythagorean theorem, this article can see that in paragraph $B D$, the movement time is:

$$
t_{B D}=\frac{\sqrt{x^{2}+\left(b-y_{0}\right)^{2}}}{v_{1}}
$$

In paragraph $D A$, the movement time is:

$$
t_{D A}=\frac{\sqrt{(a-x)^{2}+y_{0}^{2}}}{v_{2}}
$$

From (1) (2), the total exercise time is:

$$
t(x)=\frac{\sqrt{x^{2}+\left(b-y_{0}\right)^{2}}}{v_{1}}+\frac{\sqrt{(a-x)^{2}+y_{0}^{2}}}{v_{2}}
$$

To solve the problem of the minimum time required for a derivative function, and make it a value of 0 , the conditions to obtain the shortest time. Which is:

$$
t^{\prime}=t^{\prime}(x)=\frac{1}{v_{1}} \frac{x}{\sqrt{x^{2}+\left(b-y_{0}\right)^{2}}}-\frac{1}{v_{2}} \frac{(a-x)}{\sqrt{(a-x)^{2}+y_{0}^{2}}}=0
$$

The $x_{0}$ obtained at this time is the abscissa of the most advantage $D$.

Through the above-mentioned problem-solving method, it can be found that the procedure for obtaining the value simply by calculation is complicated, and if it encounters a special situation, it will not be solved. Therefore, it is feasible and effective to use MATLAB to find the coordinates of the best position.

\subsubsection{Distance}

In paragraph $B D$, the movement distance is:

$$
\begin{aligned}
& s_{1}=s_{1}(x)=|B D|=\sqrt{x^{2}+\left(b-y_{0}\right)^{2}},(0 \leq x \leq a) \\
& s_{2}=s_{2}(x)=|D A|=\sqrt{(a-x)^{2}+y_{0}^{2}},(0 \leq x \leq a) \\
& \quad s=s(x)=|B D|+|D A|=\sqrt{x^{2}+\left(b-y_{0}\right)^{2}} \\
& +\sqrt{(a-x)^{2}+y_{0}^{2}},(0 \leq x \leq a) \\
& s^{\prime}=s^{\prime}(x)=x \times\left(x^{2}+\left(b-y_{0}\right)^{2}\right)^{-\frac{1}{2}} \\
& -(a-x) \times\left((a-x)^{2}+y_{0}{ }^{2}\right)^{-\frac{1}{2}}=0,(0 \leq x \leq a)
\end{aligned}
$$

When $s^{\prime}(x)=0, s$ has the shortest distance. 


\subsection{Simulation}

\subsubsection{Simulation Process}

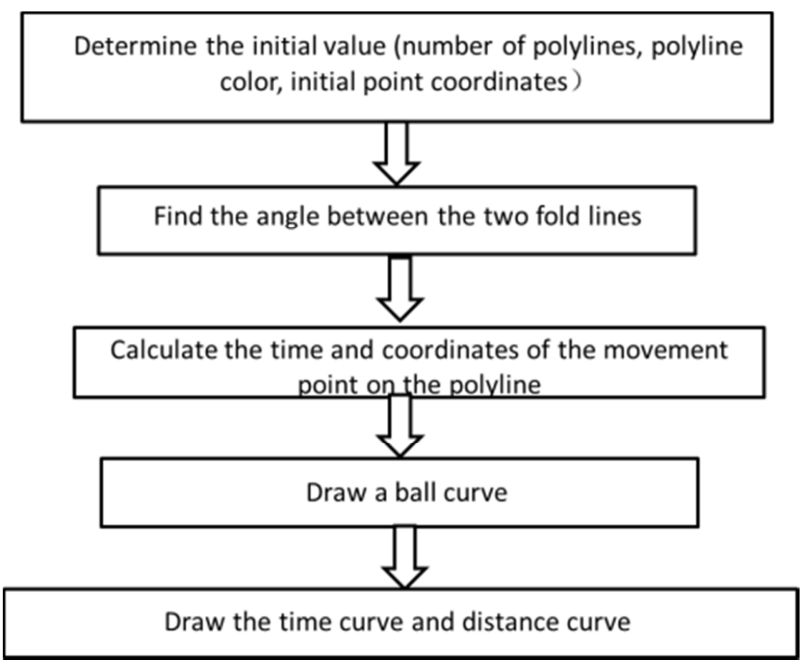

Figure 2. Simulated flow chart for the shortest time problem of constant speed drop.

\subsubsection{Polyline}

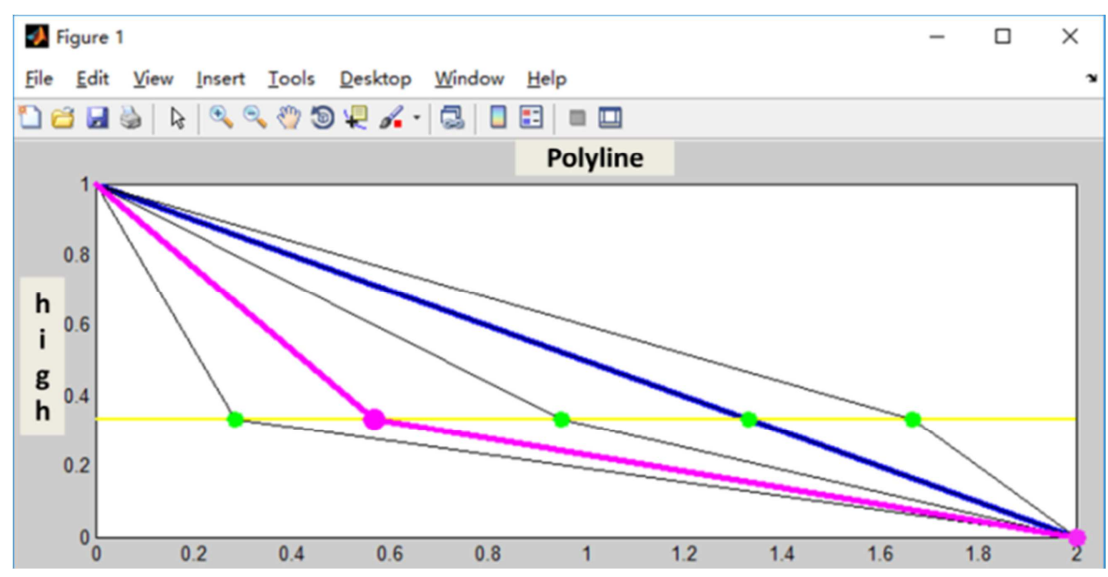

Figure 3. Polyline.

The five points in Figure 3 are taken as follows: sequentially named $D_{1}, D_{2}, D_{3}, D_{4}, D_{5}, D_{4}$ is not refracting. Follow a straight line from $B$ to $A, D_{2}$ spends the shortest time, $D_{5}$ and $D_{2}$ are symmetrical about the line of motion, $D_{3}$ is the midpoint of the $D_{2}$ and $D_{4}, D_{1}$ and $D_{2}, D_{3}, D_{4}$ are equally spaced on the left side of.

\subsubsection{Timing Schematic}

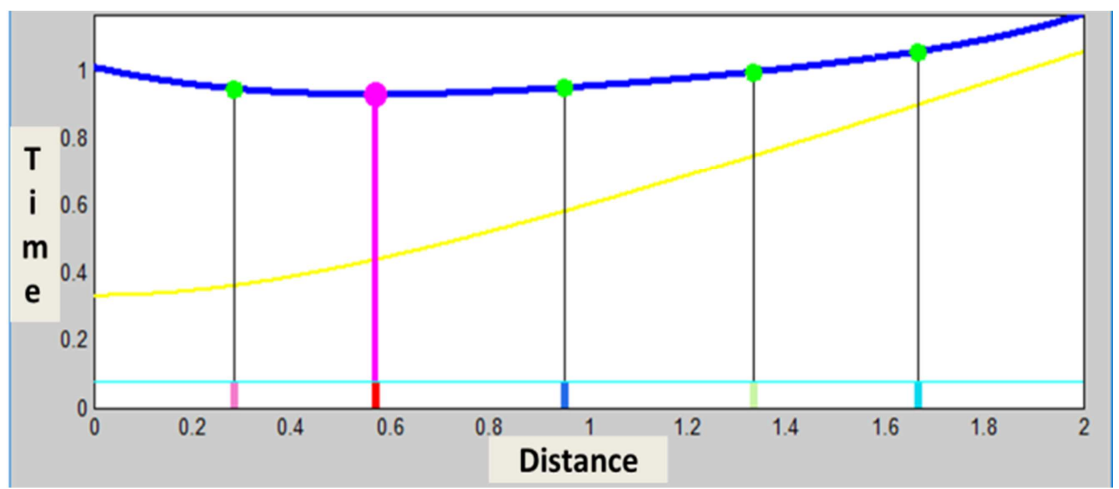




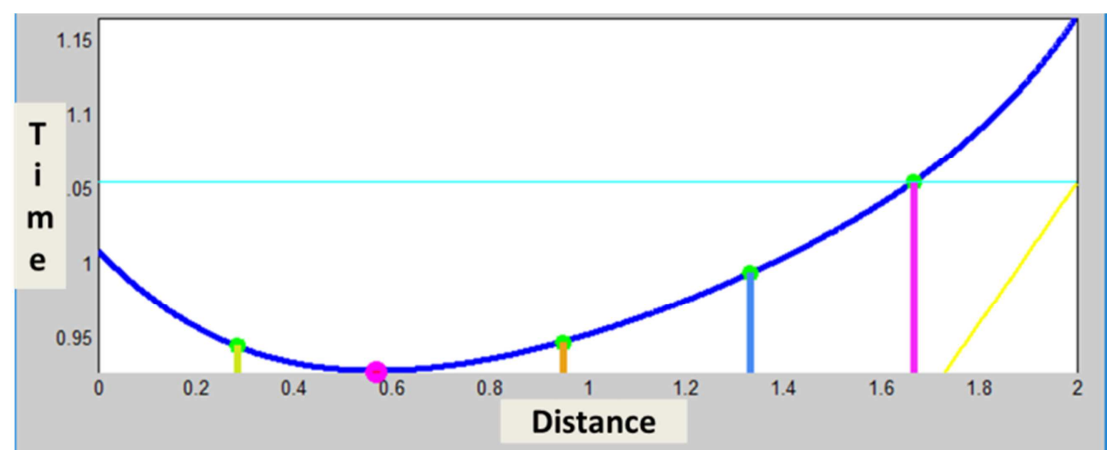

Figure 4. Motion Time Curve.

It can be seen from Figure 4 that the time-shifted ordinate corresponding to point $D_{2}$ is the smallest, indicating that the movement time of $D_{2}$ is the shortest. At the same time, based on the continuous change curve of the exercise time, the conclusion is that $t^{\prime}(x)=0$ is the point of $D_{2}$.

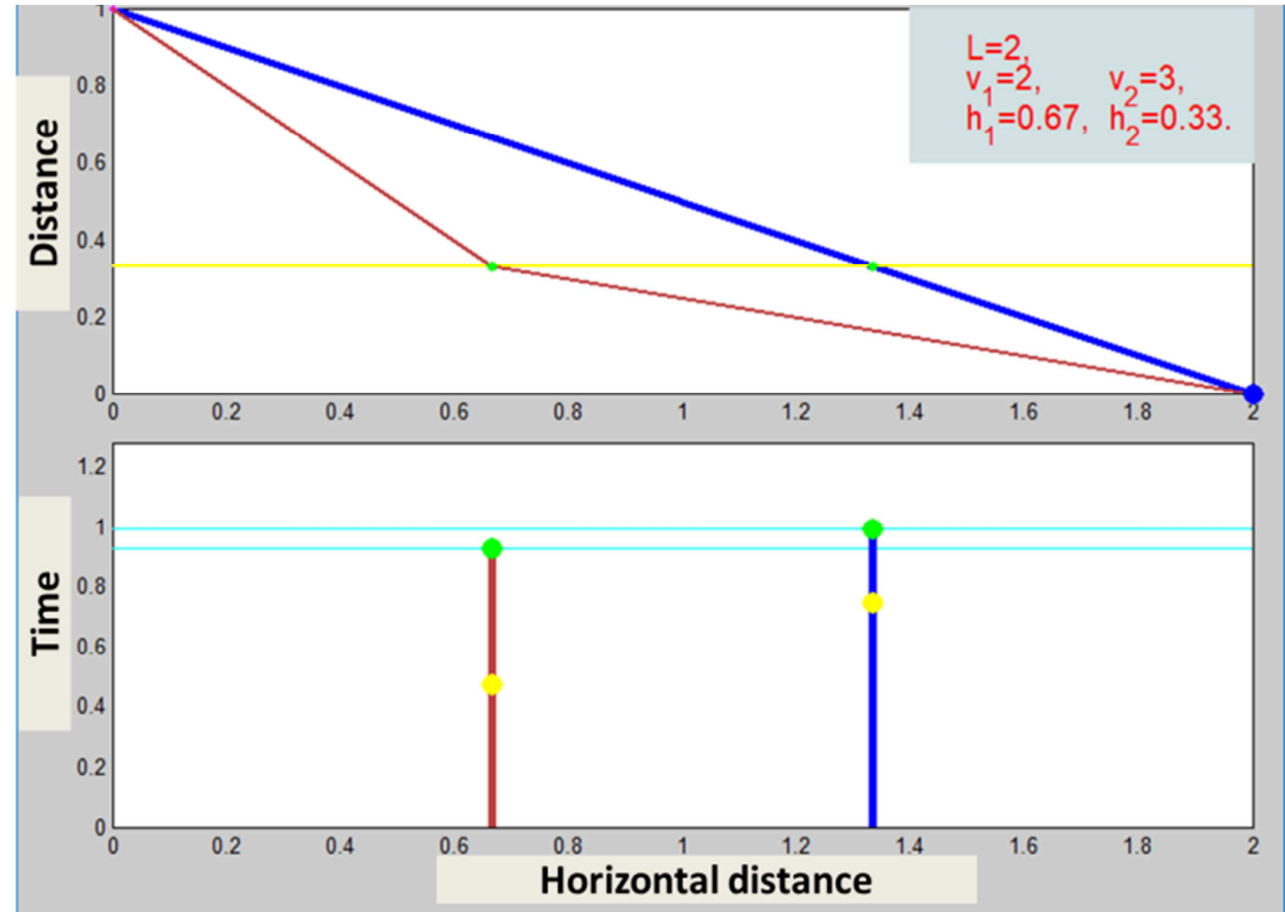

Figure 5. Sport Time Comparison Curve.

As can be seen from Figure 5, the arrival time of different points is different.

It can be seen from the results of Figure 3、 Figure 4 . and Figure 5 that due to the different angles between falling and refraction and the vertical direction, the total motion time of each scheme is different. Therefore, it can be concluded tentatively that the total movement time after $B$ reaches $A$ is related to two tilt angles $\theta_{1}$ and $\theta_{2}$.

\subsubsection{Distance Schematic}

It can be seen from Figure 6 that the shortest solution $D_{2}$ does not necessarily take the shortest distance, because in section $D A$, the movement time of $D_{2}$ is longer than that of the shortest solution. The principle can also be easily concluded.

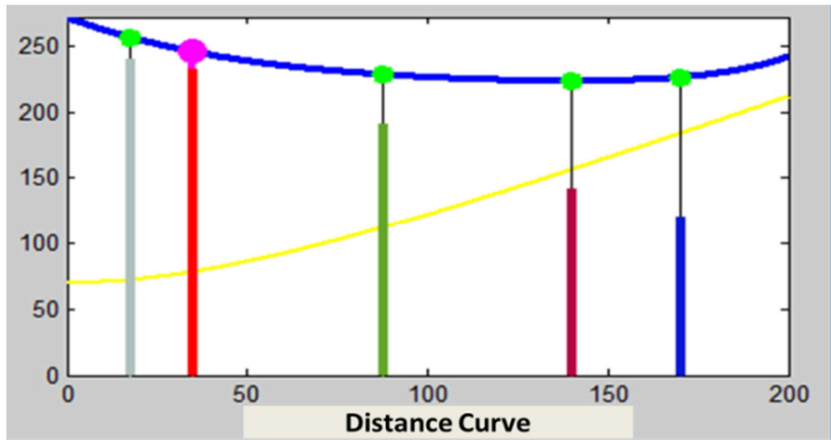

Figure 6. Sport Distance Comparison Curve.

\subsubsection{Principle Expansion}

From Figure 3、Figure 4, and Figure 5, it can be seen that 
the total time of movement through $B$ to point $A$ is related to two tilt angles $\theta_{1}$ and $\theta_{2}$. After dealing with equation (1) (2), this article get:

$$
\begin{gathered}
t^{\prime}=t^{\prime}(x)=\frac{1}{v_{1}} \frac{x}{\sqrt{x^{2}+\left(b-y_{0}\right)^{2}}}-\frac{1}{v_{2}} \frac{(a-x)}{\sqrt{(a-x)^{2}+y_{0}^{2}}}=0 \\
\frac{\sin \theta}{v_{1}}=s v_{1}(x)=\frac{x}{v_{1} \sqrt{x^{2}+\left(b-y_{0}\right)^{2}}}
\end{gathered}
$$

$$
\begin{gathered}
\frac{\sin \alpha}{v_{2}}=s v_{2}(x)=\frac{a-x}{v_{2} \sqrt{(a-x)^{2}+y_{0}^{2}}} \\
\therefore t^{\prime}=t^{\prime}(x)=\Leftrightarrow \frac{\sin \theta_{1}}{v_{1}}-\frac{\sin \theta_{2}}{v_{2}}=0 \Leftrightarrow \frac{\sin \theta_{1}}{v_{1}}=\frac{\sin \theta_{2}}{v_{2}}
\end{gathered}
$$

This article can see that when $\frac{\sin \theta_{1}}{v_{1}}=\frac{\sin \theta_{2}}{v_{2}}$, this article can get the shortest time. The following is the simulation visualization result:

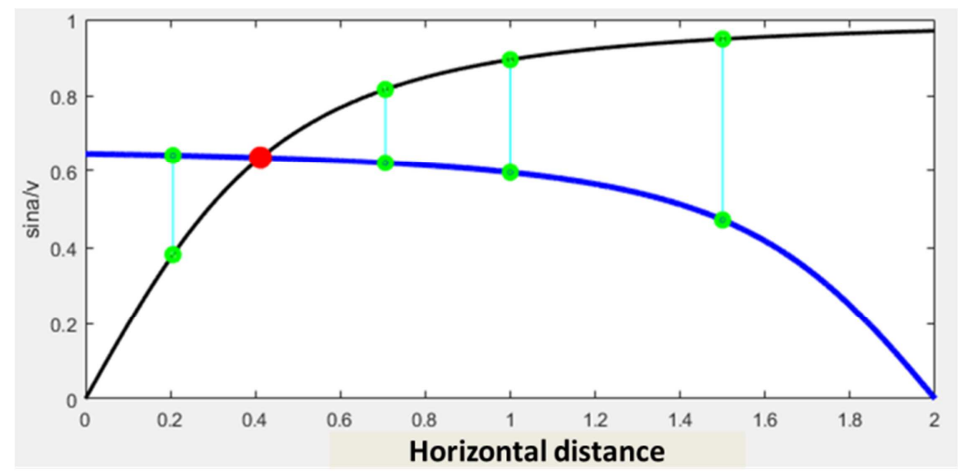

Figure 7. Ratio Curve.

Figure 7 shows that at $\frac{\sin \theta_{1}}{v_{1}}=\frac{\sin \theta_{2}}{v_{2}}$, the corresponding route has the shortest movement time. At the point on the left side of $D_{2}$, when it is at segment $B D$, the exercise time is shorter than $D_{2}$, and when it is at segment $D A$, the exercise time is longer than $D_{2}$; At the point on the right side of $D_{2}$, when it is at a section $B D$, the exercise time is shorter than that at case $D_{2}$, and when it is at a section $D A$, the exercise time is shorter than $D_{2}$.

\subsubsection{Dynamic Presentation Process}

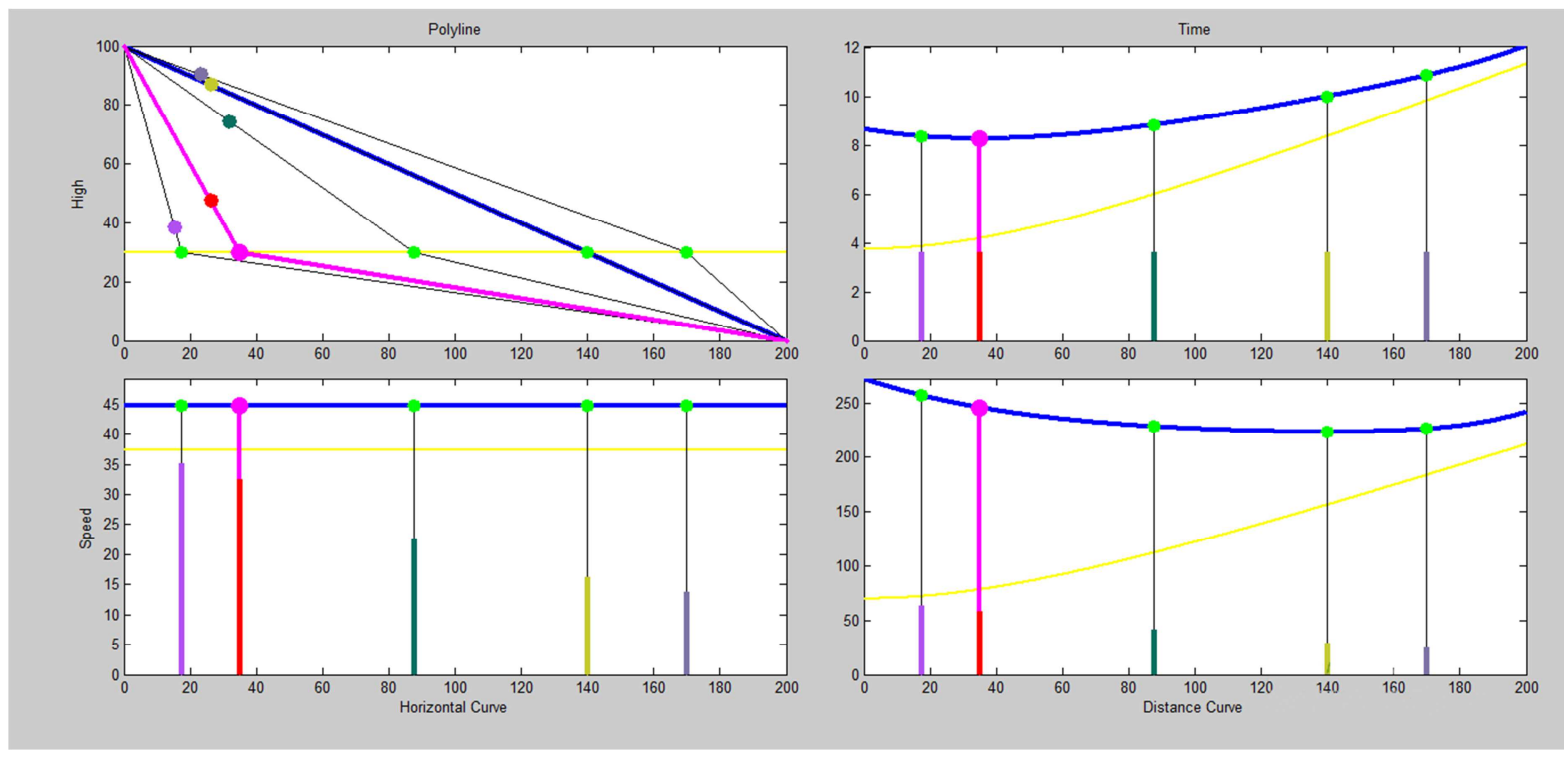

Figure 8. The Movement of the Refraction Plane 1.
In addition, find the second derivative of $t(x)$ :

$$
\begin{aligned}
& t^{\prime \prime}(x)=\frac{1}{v_{1}}\left[\frac{\left(b-y_{0}\right)^{2}}{\left(x^{2}+\left(b-y_{0}\right)^{2}\right)^{\frac{3}{2}}}\right]+\frac{1}{v_{2}}\left[\frac{y_{0}{ }^{2}}{\left((a-x)^{2}+y_{0}{ }^{2}\right)^{\frac{3}{2}}}\right] \\
& >0
\end{aligned}
$$

It's proving that $\frac{\sin \theta_{1}}{v_{1}}=\frac{\sin \theta_{2}}{v_{2}}$ is the only minimum point. 
As can be seen from Figure 8, the movement distance of $D_{1}, D_{2}, D_{3}, D_{4}, D_{5}$ increases sequentially in the $B D$ segment, and the time required to pass $B D$ increases in the same speed. It can be seen from Figure 8 that due to the segment $B D$, the movement distance in $D_{1}, D_{2}, D_{3}$ is shorter than $D_{4}, D_{5}$, so $D_{1}, D_{2}, D_{3}$ leads into refraction first.
In paragraph $D A$, the distance of $D_{1}, D_{2}, D_{3}, D_{4}, D_{5}$ travelled in descending order. Therefore, compared to $D_{2}$, the required time of $D_{1}$ for exercise increases, which explains why the following $D_{2}$ is shorter than $D_{1}$.

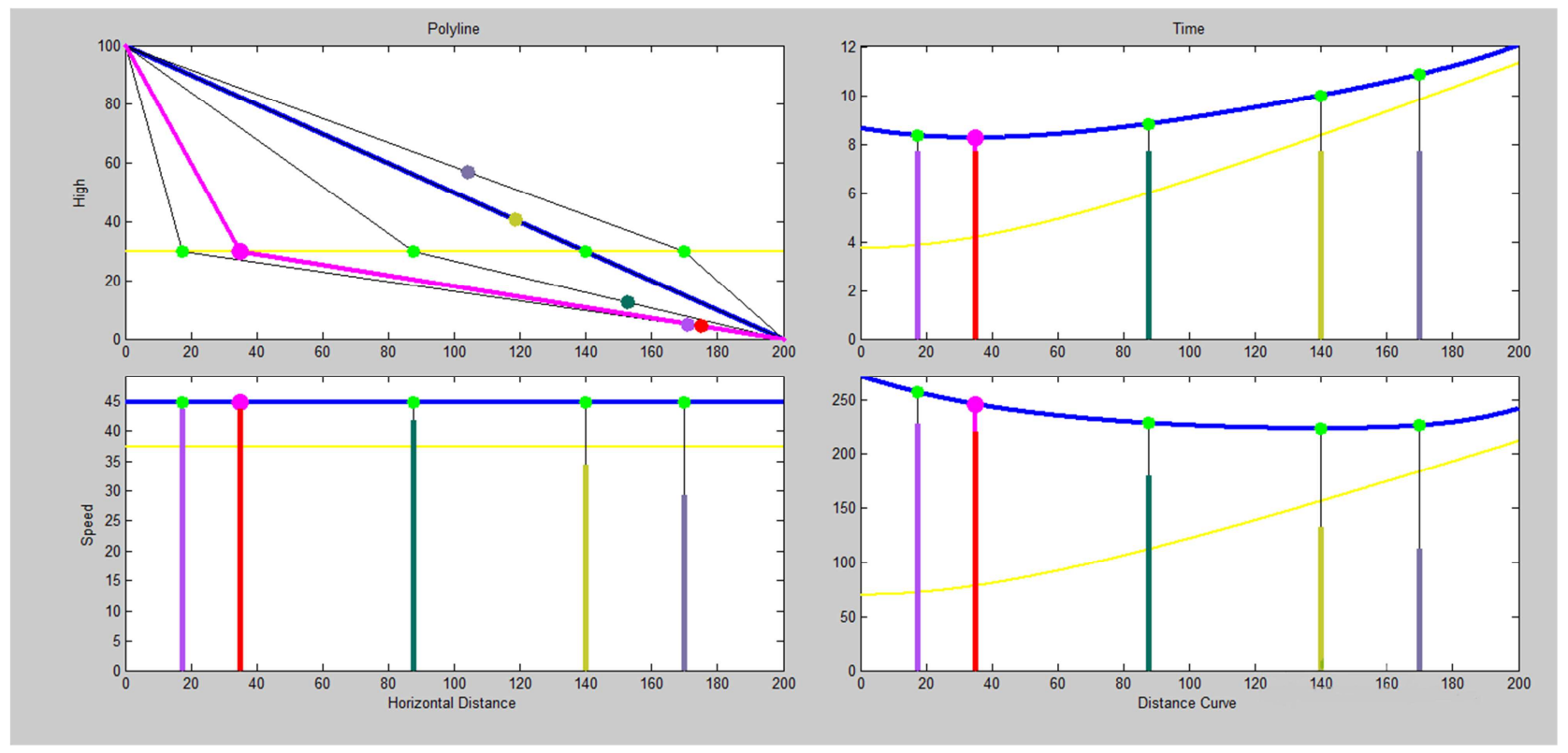

Figure 9. The Movement of the Refraction Plane 2.

It can be seen from Figure 9 that in the segment $D A$, the movement distance decreases in turn and the required movement time of $D_{1}, D_{2}, D_{3}, D_{4}, D_{5}$ decreases in turn. Therefore, $D_{2}$ reaches the end point earlier than $D_{1}$. Through rigorous theoretical calculations, it can also be shown that

When $\frac{\sin \theta_{1}}{v_{1}}=\frac{\sin \theta_{2}}{v_{2}}$, a total exercise time is indeed the shortest. This dynamic simulation solves this problem well.

In the dynamic simulation, the movement of each point can be easily observed. You can see the movement time of each broken line and the comparison chart of the distance traveled. Compared with the simple theoretical calculations, simulations achieve better observation and actual results.

\section{The Shortest Time for Uniform Acceleration and Descent}

\subsection{Problem Description}

The particle moves from point $B(0, b)$ to point $A(a, 0)$, the speed of line $B D$ is $v_{1}$, and the speed of line $D A$ is $v_{2}$. Find the horizontal axis $x$ of the point $D\left(x, y_{0}\right)$ on the horizontal line $y=y_{0}$, so that the time from $B$ to $D$ reaches $A$ is the shortest. The difference between them and the uniform motion is that the two speeds are different. In the acceleration movement, the speed is treated as the average speed at both ends of the free fall. As shown in Figure 1.

\subsection{Solution Analysis}

First of all, according to the physical formula of free fall, the velocity of the particle whose initial velocity is zero falls to the point $D$ (slope direction) is $v_{D}=\sqrt{2 g\left(b-y_{0}\right)}$, and the average velocity from point $B$ to point $D$ can be obtained as:

$$
v_{1}=\frac{v_{D}}{2}=\frac{\sqrt{2 g\left(b-y_{0}\right)}}{2}
$$

The time from point $B$ to point $D$ is:

$$
t_{1}=t_{1}(x)=\frac{|B D|}{v_{1}}=\frac{\sqrt{2} \sqrt{x^{2}+\left(b-y_{0}\right)^{2}}}{\sqrt{g\left(b-y_{0}\right)}}
$$

Similarly, the speed at which the particle drops to point $A$ is $v_{A}=\sqrt{2 g b}$, so the average speed from point $D$ to point $A$ is:

$$
v_{2}=\frac{v_{D}+v_{A}}{2}=\frac{\sqrt{2 g\left(b-y_{0}\right)}+\sqrt{2 g b}}{2}
$$

The time from point $D$ to point $A$ is: 


$$
t_{2}=t_{2}(x)=\frac{|D A|}{v_{2}}=\frac{\sqrt{2} \sqrt{(a-x)^{2}+y_{0}^{2}}}{\sqrt{g\left(b-y_{0}\right)}+\sqrt{g b}}
$$

After the above inference, it can be obtained that the entire period of time is:

$$
t(x)=\frac{\sqrt{2} \sqrt{x^{2}+\left(b-y_{0}\right)^{2}}}{\sqrt{g\left(b-y_{0}\right)}}+\frac{\sqrt{2} \sqrt{(a-x)^{2}+y_{0}^{2}}}{\sqrt{g\left(b-y_{0}\right)}+\sqrt{g b}}
$$

Since the average velocity $v_{1}, v_{2}$ of the two segments is only related to height, not related to point $x$ on the horizontal line $y=y_{0}$, the derivatives of $t$ are:

$$
\begin{aligned}
& t^{\prime}=t^{\prime}(x)=\frac{1}{v_{1}} \frac{x}{\sqrt{x^{2}+\left(b-y_{0}\right)^{2}}}-\frac{1}{v_{2}} \frac{(a-x)}{\sqrt{(a-x)^{2}+y_{0}^{2}}} \\
& =\frac{\sin \theta_{1}}{v_{1}}-\frac{\sin \theta_{2}}{v_{2}}=0
\end{aligned}
$$

It can be observed that when the angle and velocity satisfy the law of refraction, that is, when $\frac{\sin \theta_{1}}{v_{1}}=\frac{\sin \theta_{2}}{v_{2}}$ is satisfied, the time spent is the shortest.

It should be noted that the evaluation here can be obtained by using the dichotomy method to solve the problem of $t^{\prime}=t^{\prime}(x)=0$, or the dichotomy method can be used to solve the problem of $\frac{\sin \theta_{1}}{v_{1}}-\frac{\sin \theta_{2}}{v_{2}}=0$. There are many kinds of solving methods, and the former is used for simulation.

\subsubsection{Result Analysis}

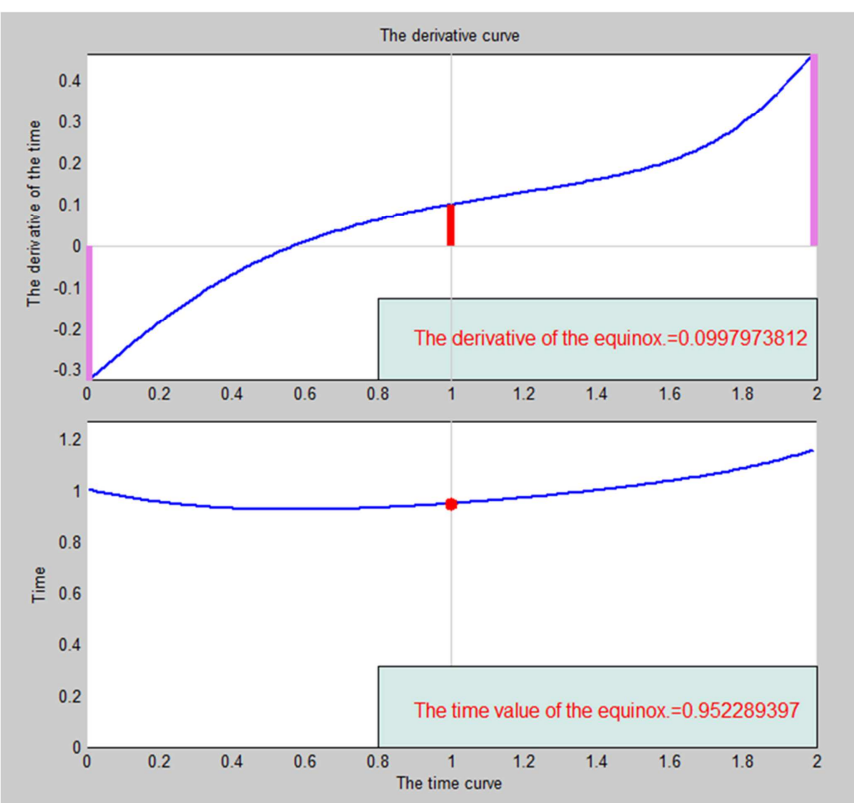

\subsection{Simulation}

In the uniform motion, the extreme points can be directly obtained, but because the evaluation method to be used in the uniform acceleration motion is more complicated, this paper considers using the dichotomy idea to solve.

The dichotomy idea mainly consists of: for a function that is continuous and $f(a) \times f(b)<0$ on the interval $[a, b]$, by continuously dividing the interval where the zero of the function $f(x)$ is in two, so that the two endpoints of the interval gradually approach zero, and then the method of obtaining the zero point approximation is obtained.

\subsubsection{Simulation Process}

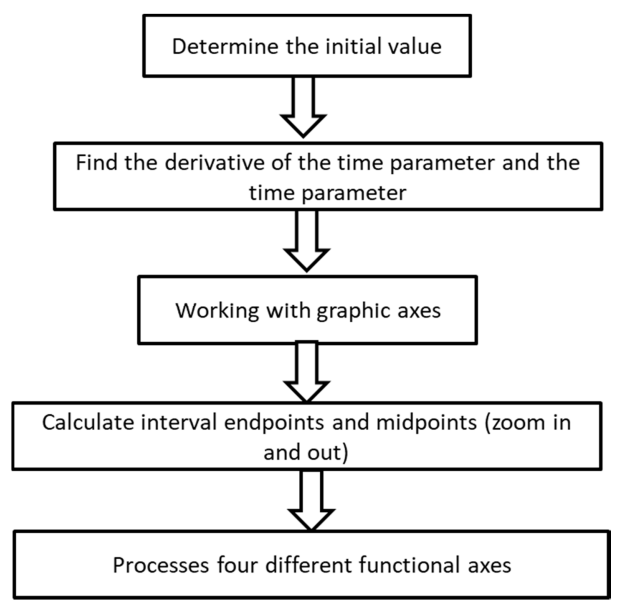

Figure 10. Simulated flow chart for the shortest time problem of uniform acceleration and descent.

Figure 11. Dynamic simulation of the shortest time problem of uniform acceleration and deceleration 1. 
Figure 11 is to draw the time derivative curve, select endpoints, use the nature of the bifurcation point to find the next point coordinates; In the second figure to enlarge the observation point; The third figure can be seen in the time value changes; The four figures can be seen as an enlarged view of the time value.
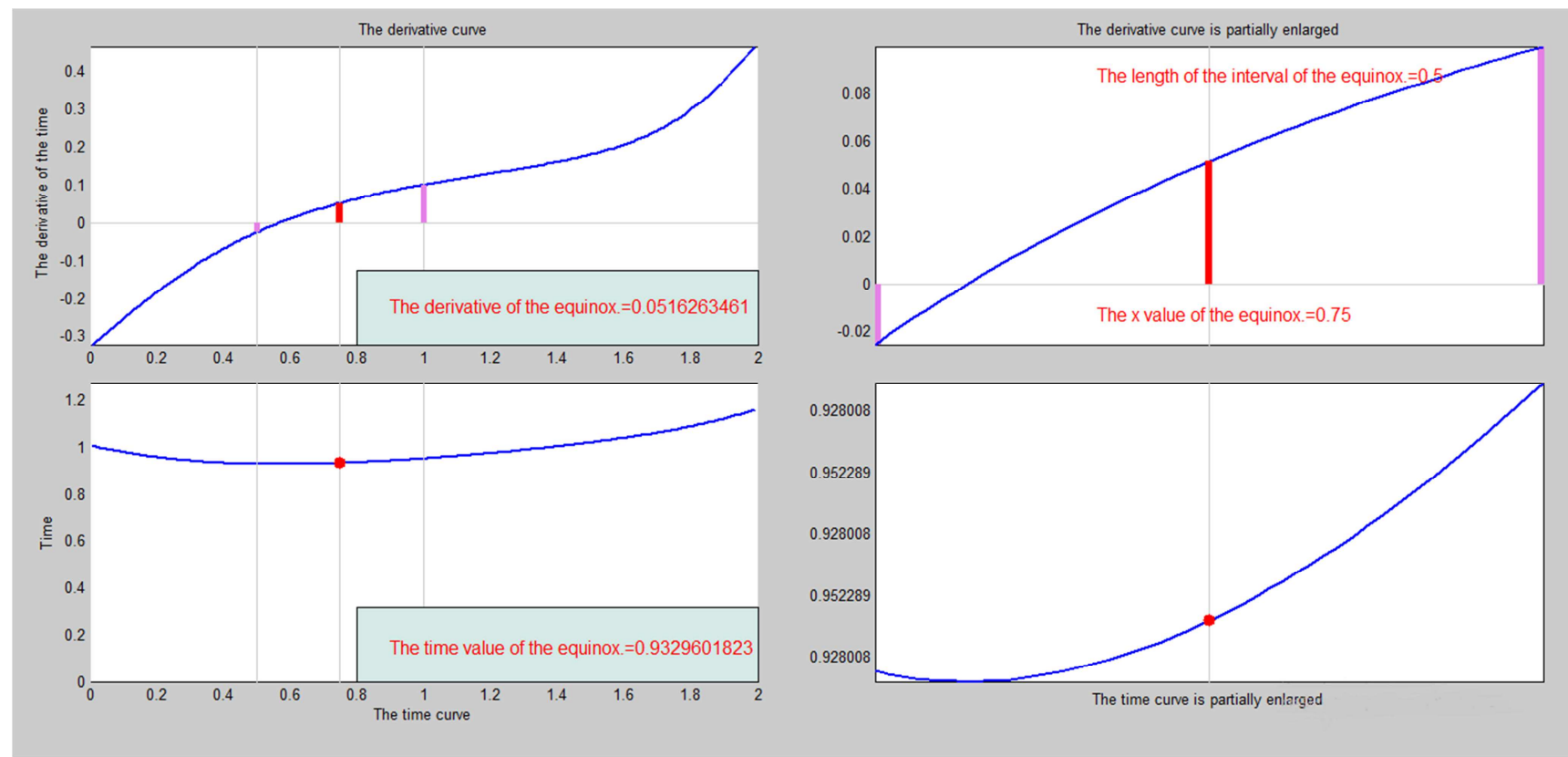

Figure 12. Dynamic simulation of the shortest time problem of uniform acceleration and deceleration 2.

Figure 12 shows the process of the third iteration. It can be seen that the derivative value is approaching zero and the time curve is continuously approaching the minimum point.
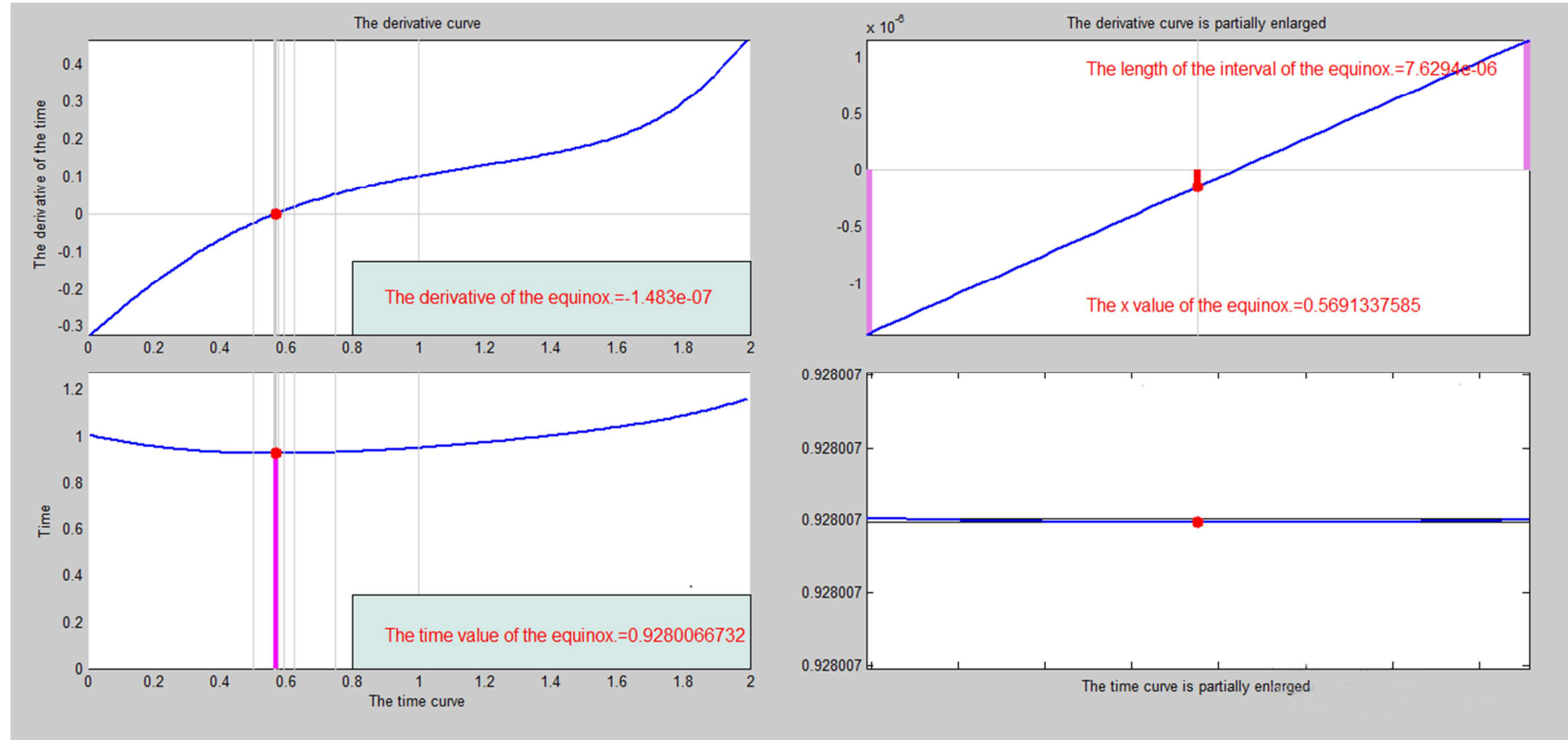

Figure 13. Dynamic simulation of the shortest time problem of uniform acceleration and deceleration 3.

Figure 13 shows the last iteration. It can be seen that the derivative of the final result is close to 0 , and the last time value is very small.

\subsection{Extension}

It should be noted that the uniform acceleration motion can be generalized to any speed. If the speed is $V=V(x)$, in any interval $\left[z_{1}, z_{2}\right]$, the average value in that period of time can be calculated through integration. This provides a solid foundation for future complex calculations 


\section{The Comparison of the Uniform and Uniform Acceleration Movements}

\subsection{Spatial Thinking}

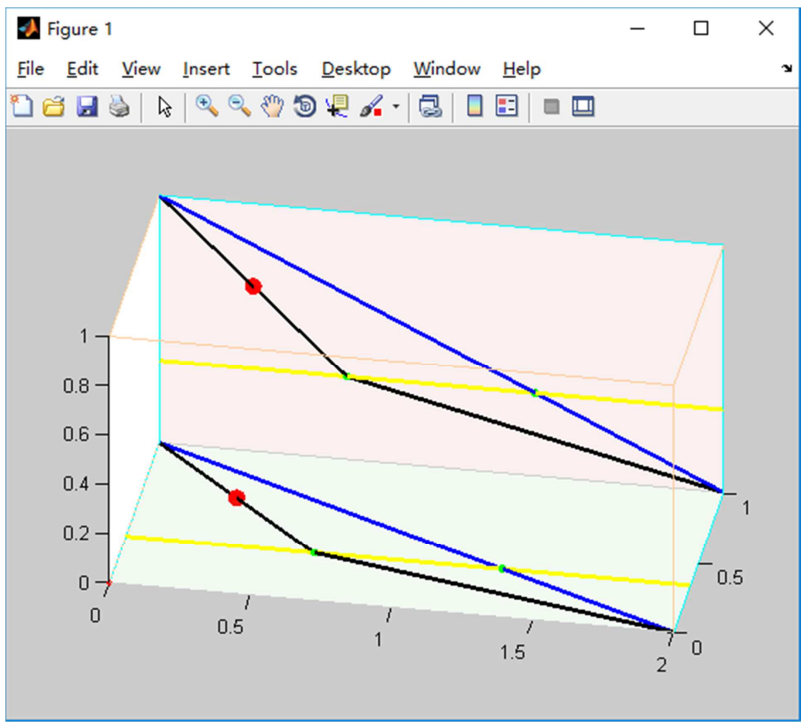

Figure 14. The comparison of the Uniform and uniform acceleration movements in the space.

The uniform motion and the uniform acceleration motion can be classified into different spatial conditions. The speed of uniform motion will not change, and the speed of uniform acceleration motion will change with the acceleration of free fall. Therefore, this paper will increase the space $\mathrm{Z}$ axis by uniformly accelerating motion. On the changes, the graph can more clearly distinguish the relationship between uniform motion and uniform acceleration.

\subsection{Extension Issues}

The problem of the shortest time for uniform and accelerated descent can be obtained:

When $\frac{\sin \theta_{1}}{v_{1}}=\frac{\sin \theta_{2}}{v_{2}}$ is satisfied, the time from $B$ to $A$ is the shortest. This problem can also be extended to the problem of uniform acceleration of multiple lines, that is, when $\frac{\sin \theta_{1}}{v_{1}}=\frac{\sin \theta_{2}}{v_{2}}=\ldots=\frac{\sin \theta_{n}}{v_{n}}$ is satisfied, the total motion time is the shortest. Therefore, the steepest descent line is a condition that satisfies $\frac{\sin \theta}{v}=C \quad(\mathrm{C}$ is a constant $)$.

\section{Conclusion}

The MATLAB simulation results can clearly see the time and location of the steepest descent point. Whether it is uniform or uniform acceleration, the following conclusions can be drawn: Point $D\left(x, y_{0}\right)$ from left to right from the horizontal position, the total time used first decreases and then increases, there is a minimum value;
When the curve $\frac{\sin \theta_{1}}{v_{1}}$ intersects with the curve $\frac{\sin \theta_{2}}{v_{2}}$, it is the shortest point $D$ for application; the best point $D$ 's exercise plan is not the shortest route. The dichotomy is a better way to find the extremum problem. Although the declining problem is simple in description, it contains the shortest time and the shortest distance thoughts. It is also a subject worthy of study to combine various extreme values.

\section{Acknowledgements}

This article is funded by the Beijing University of Science and Technology Education Research Project (JG2017Z10).

\section{References}

[1] Xue Ting. Some Thoughts on the Extremum Method of One-way Function [J]. Exam Weekly. 2011 (52).

[2] Sheng Zhaobiao. Basic tutorial on optimization method [M]. Nanjing: Southeast University Press, 1992.

[3] Li Xiufen, Wang Pinghua, Liu Jiang, Jin Lei, Ruan Tongbin, Sun Yingjie, Peng Feng, Pan Yuhong. Precipitation extremum characteristics of eastern Liaoning during 1960-2015[J]. Journal of Ecology. 2017 (08).

[4] Shi Xianwu, Fang Weihua, Lin Wei, Li Ying. Analysis of Uncertainty in Precipitation Distribution in China Based on Extreme Value Theory [J]. Journal of Beijing Normal University (Natural Science); 2011 (05).

[5] Yi Zhaohua, Huang Tianyi. The Problem of the Shortest Time Orbit for Launching Cosmic Rockets to Mars [J]. Yi Zhaohua, Huang Tianyi. Astronomy Journal. 1961 (Z1).

[6] Xie Chengwang, Hu Jiabao. Extremum problem solving based on genetic algorithm [J]. Journal of Wuhan Institute of Science and Technology. 2004 (02).

[7] Liu Jian, Yu Jianning. Models and Algorithms for Multimodal Transport Mode Selection [J]. Journal of Lanzhou Jiaotong University. 2010 (01).

[8] Zhang Jun, Han Zhengbao. Multi-modal transport mode selection based on minimum total cost $[\mathrm{J}]$. Value Engineering. 2014 (08).

[9] Wang Jianqiang, Yang Chin. Research on the Coupling Degree Model of Multiple Transport Modes for Comprehensive Transport Channels [J]. Journal of Lanzhou Jiaotong University. $2016(06)$.

[10] Duan Xiaohong, Zhao Jiandong, Song Shouxin. Dynamic Shortest Path of Road Network Emergency Vehicle Based on Hybrid Leapfrogging [J]. Transportation Systems Engineering and Information. 2016 (03).

[11] Wang Chunyuan, Liu Shuhui. The shortest path model and solution for robot walking obstacle avoidance in given scene [J] Silicon Valley. 2013 (16).

[12] Yu Haibo. An example of the solution to the problem of physical extremes [J]. Literacy navigation (middle). 2011 (07).

[13] Feng Kejiang. Several Solutions on Extreme Value Problems [J]. Journal of Qinghai Teachers College. 1988 (03). 
[14] Lin Zhili, Zhu Daqing, Pu Jixiong. MATLAB animation technology research in visual teaching of electromagnetic theory curriculum $[\mathrm{J}]$. China Modern Education Equipment. $2017(03)$

[15] Zhou Jian, Jin Jian, Liu Daojun, Cheng Dong. Application of Matlab simulation in Helmholtz coil magnetic field measurement experiment [J]. Journal of Wanxi University. 2017 (02).

[16] Wang Jie. Study on the Teaching Reform of Electrostatic Field Based on Matlab Simulation and Computation [J]. Educational Modernization. 2016 (01).
[17] Li Bangxing. Mathematical physics method for realization of the MATLAB visualization of the formula of Dalembert [J]. Scientific Consulting (Technology/Management). 2017 (08).

[18] Jiao Zhilian. Application of MATLAB in Mathematical Physics Method [J]. Software and Application of Computer Optical Disk. 2014 (20). 